\title{
GARMENTS UNDER BRACE LIFE QUALITY IMPROVE AFFECT EXPERIMENT OF TECHNOLOGY AND PHYSIOLOGY
}

Orsolya Nagy Szabó, András Koleszár

Óbuda University, Faculty of Light Industry and Environmental Engineering szabo.orsoby@rke.uni-obuda.bu

\begin{abstract}
Scoliosis is a spinal deformity that most commonly appears in girls and makes itself apparent at puberty during the early teenage years. Living with scoliosis can be especially difficult for children, particularly when a brace is required to stop its progression. The context of clothing physiology help a clothing system of air, vapor permeable insulation and opportunities. These same characteristics are changed when brace wearing, but also help the right materials and design concepts for the development. In view of the currently available properties of fabrics during the test and we see clothing physiology using different raw materials and functional textiles, as well as the solution for improving the body's microclimate.In our research achieve an optimal physiological state of body with a corver on the brace,that improves the body's microclimate, reduce the unpleasant effects of thebrace at high temperatures, but the basic function of the medical device support.
\end{abstract}

Keywords: Scoliosis, brace, underwear, clothing physiology.

\section{Introduction}

12 million people suffer some form of scoliosis all over the world. ${ }^{1}$

There are 450 new scoliosis cases each year in our country, but among them 150 patients need orthopedic help, but the number is steadily increasing. ${ }^{2}$

There are many types of scoliosis. Depend on the disease type and severity of various types were made brace in custom size.

Orthopedic surgeon diagnose spinal disease and prescription a brace, according to the type of the spine disease, and the brace is made of by the orthopedic technician.

Conservative treatment of diseases the $5 \mathrm{~mm}$ thick plastic "armor" should be weared, which can only be effective if the 24-hour of a day at least 18-20 hour wear the child until they beome adult.

Our goal is to develop a system of clothing that is worn under brace and in addition to improving the level of comfort in wearing and reduced uncomfortable feeling of brace.

The brace is not only caused a physical hardship but also it has very negative effect on young people.

The proper set up and wearable of brace associated with comfort feeling the clothing which is worn under, the comfort and aesthetic properties should be improved.

The fabrics currently available, taking into account the properties of the test during the brace worn which cause a variety of clothing physiology problems based on functional textile underwear. Our research will seek a solution to improve the body's microclimate. 
The aim is to achieve an optimal physiological conditions with underwear or a special pulled coating product development that improves the body's microclimate, reduce unpleasant effects of the device, but the basic function of a medical device supports.

We desiged some underwear products based on children needs: T-shirts, bras, panties, pulled clothes to brace and with construction, modeling, technology implementation we have tried to satisfy all those requirements, which are adapted to this special wearability.

We have developed some modell variation and we selected functional fabrics with special properties, eg. Antibacterial Coolmax, Outlast PCM and flexible cotton, and we tested this tshirts from tis special textiles.

The underwear design process greatly affect the wearing comfort. The material properties are taken into account - which are based on various textile testing - we tried to identify additional opportunities with which to improve our children's wellbeing.

\section{Methods and materials}

\subsection{The commercially available underwear products}

Commercial cotton shirts do not follow the body shape, and thus under the brace crinkles in which prolonged skin irritation. There are variety of sportswear specifically designed and made of special textile T-shirt, underwear available. These are not suitable because:

- The location of cutlines are depend on fashion, it does not take into account the pressure points of the brace,

- T-shirts are typically in the form of body shape but they have too many cutlines

- The seams are not always lapped seam

- Construction of the T-shirts is not precisely follow the body shape

- For many of the brace wearer the cutline through the armpits specific irritant

- From functional materials fashion sportswear products are expensive

- Seamless, form knitted T-shirts are also available but these textiles made of polyester, which is in is not comfortable to wear, and the knitted pattern is pressed into the skin.

- The operating instructions and the size designation is designed for comfortable

\subsection{Problems under wearing brace}

Upper body movement is very limited in brace it can not bend down, turn right and left is limited.

The two most important features of the clothing are the thermal insulation and water vapor permeability. They affect thermoregulation of human body.

The tests are performed in some various designs and textiles underwear, and the brace had and old and an improved version was performed in which the air and leak tightness can be improved by means of perforation. 
We had a survey among children who carried out brace. Based on the answers to most of their distractions while they were wearing brace.

- There is the pressure in armpit

- Rub the skin in pressure points

- Crumpled shirt, slide up underneath the dress

- Do not wear when in a less visible from the outerwear

- Placing bra clips

\subsection{Physiological effect}

The context of clothing physiologic give help a clothing system air and vapor permeable insulation and optimum adjustment possibilities. Problem is that these same indicators change when the brace is worn. The method helps to select the appropriate materials and design concepts and develop the textile selection. The clothing thermal insulation and in the brace composition of the material to be tested due to the different temperatures of the body "comfort". Materials is to look at under brace in which improve the physiological conditions, and a built-in intelligent systems to be develop and improve comfort feeling.

Two most important characteristics of the clothing thermal insulation and water vapor permeability. They affect human thermoregulation. We make various tests based on underwear design and tailoring, as well as at present and in an improved version of brace wich has air through perforations and features of brace can be improved.

It should be noted the problem at the pressure points of the brace and the body relationship which may affect the size of the holes in the skin bulges, which can cause permanent damage.

\subsection{Criteria for selection of appropriate materials}

A functional and smart textiles, which are due to their composition, or as a result suitable for a finish to protect health, improve quality of life. In our study, we were looking for answers to the new generation of commercially available materials which parameters in test conditions, which best meet the needs of customers physiology.

Our basic requirements from textiles:

- Good air permeability

- Antibacterial

- Moisture-wicking

- Good abrasion resistance

- Washable

- Good to be processed. 
Many different compositions materials were tested and tried in sports activities / walking, running, ice- hockey, / and wearing brace.

A further studies of the best properties of materials continued.

The wearing of cotton-type tests based on the subjective evaluation of Outlast and Coolmax functional fabrics selected from the raw material of T-shirts.

\subsection{Major features of materials}

\section{Cotton}

Cotton is a soft, fluffy staple fiber that grows in a boll, or protective capsule, around the seeds of cotton plants of the genus Gossypium.

The cotton fabrics have soft and high breathability properties. Good hygroscopic, deep in water it can be taken 25 to 27 times up their own weight. It is difficult to dry and does not charge electrostatically. The fabric quality depends on the quality of the cotton fibers. ${ }^{3}$

\section{Outlast}

At the textiles also used special particles in the liquid-crystalline materials, changes in the physical state of the unusual phenomena can be observed:

- The melting of solid phases at the first one for liquid, dense, confusing, "liquid crystal" state,

- Followed by further heating the isotropic liquid and gaseous materials.

A "normal" liquids isotropic property of the liquid-crystalline state, however, the material coming from different directions and respond differently influences ("anisotropic" phase). The "Phase Change Material," the phrase in English PCM-agent spread after phase-alternating phasechanging, state-set exchange properties. The PCM-s ability to absorb a significant amount of heat energy, temporarily stored and then adapting to the changes in the environment of this latent heat is utilized. If temperatures in the range of their physical state change request:

- Solid phase near liquid state is cooling,

- Liquid cooling states of matter has an external effect, e.g. heat loss. The PCM is stopped before the melting point of the heating cycle. ${ }^{4}$

That Outlast technology, the company was first developed for astronauts the basic materials of everyday life can be used in many fields, especially for clothing.

\section{Coolmax}

COOLMAX fabric with a specially designed polyester fiber made from elemental that wicks away perspiration from the body, and through the material quickly evaporates, so the clothing wearer comfort improvement. Figure 5 has shown 4 and 6-channel fibers develop. The increased fiber surface due to the tissue surface of the water quickly evaporates. ${ }^{5}$ 


\section{Results and discussion}

\subsection{Underwear design point of view}

The design takes into account the material properties and the specific physiological needs, which arise for the corset wearers. Primary consideration when designing products in the seam, relocation, removal armpit seam, and the less cutline longitudinal design. A "hard armor" is one feature of the wearer's armpits to push, helping to maintain proper spine.

Problems during wearing brace:

- The pressure points at the skin becomes red,

- Underwear seams deeply press in the skin

- In armpit strong pressure and rubbing occurs,

- Commercially available products underwear cause wrapping problem and t-shirt slide up under brace

- The body sweat more strongly especially during movement

- Summer weather conditions, the wearing comfort is extremely bad.

\subsection{T-shirt design}

1. Brace pressure in armpit

2. T-shirt don't wrap under brace

3. Neck curve cutline delete in armpit

t-shirt construction with special method

don't see from upper clothes

Taken into account the area of the most commonly used pressure points of the brace, which was specifically designed for the T-shirt.

The t-shirt is made of a flexible textile, depending on the body size, and in the construction need $5-25 \%$ below in the wrap direction and $5-10 \%$ rod direction to prevent the formation of wrinkles on wearing, which rub the skin. This greatly increases the comfort feeling.

Taking into account we designed the cut lines of the t-shirt most ideal locations different way of usual cut lines.

First of all 3 kinds of cutlines placement was planned, taking into account the development of medical devices typical pressure points consultation with orthopedic technician, so the shirts are comfortable in wearing the seam widths pressed in the skin minimal.

Take into account a variety of cutline shirt was designed everyone can find right in which the seam pressure does not irritate the skin.

The pattern size can be made manually, based on the purchase, preparation of computer manufacturing system and 3D body scanners. The pattern should be considered in the design of pressure 


\subsection{T-shirt descrition}

T-shirt is tight, closely following the body. The length of it is ending in hip line, but it is variable. Slives can be made of short and long, raglan, Japanese and normal version. Neck curve can be round or "V"-cut. Cutlines primarily depends on pressure points on, at girls / women / it is formatting breast.

Some of the possible types of product plan (Figure 1.):
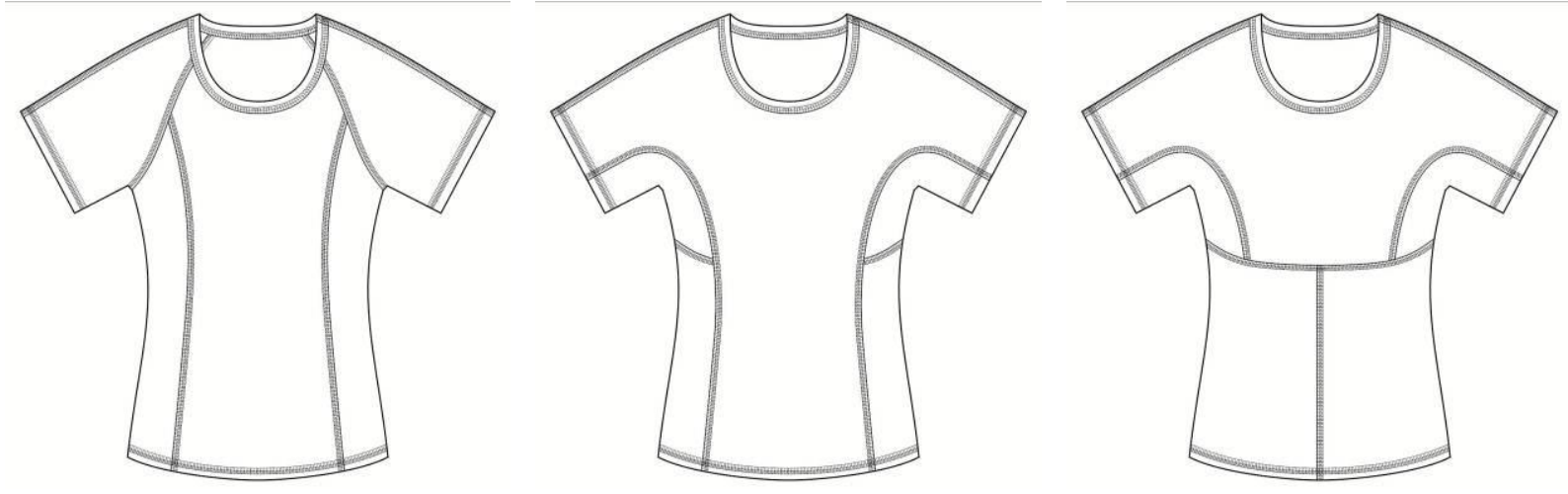

Figure 1:T-shirts

\subsection{T-shirt technology}

The technology is making 3 or 4 pin coverstich sewing machine with lapped seam. One possible solution is that all cutlines use this kind of seam technology and decorations can result in the use of colored thread.(Figure 2)

The assembly sequence of operations depends on the location of the cut lines.
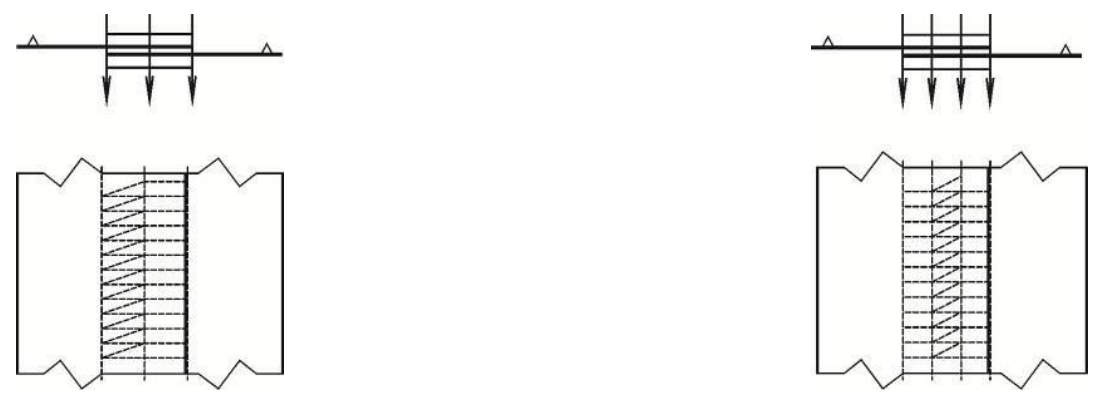

Figure 2: 3 or 4 pin coverstich sewing 


\subsection{Bra and slip design}

The wearer of brace is a higher percentage of young women who are already wearing a bra. This is a problem for many people because of one pressure points is definitely a place where the brace has a pressure point and bra back buckle or adjuster is located.

We designed a bra that buckle is at the front, and the special rubber does not cause skin irritation under brace. The cups of bra can be made of cut lines (see Figure 3), but without cut lines thermoforming and foam version. The bra can be made of underwire if it is not cause pressure in skin. If this solution does not work, be prepared for a variation of built-shirt bra.
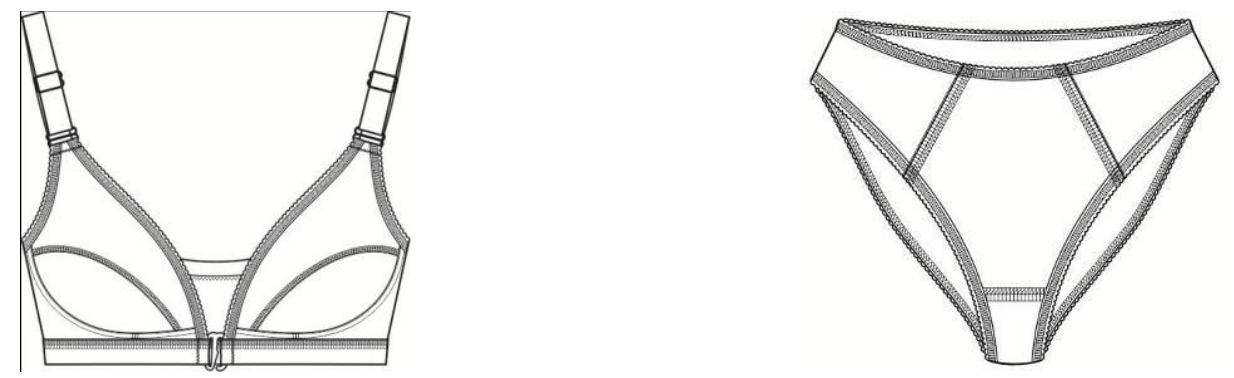

Figure 3: Bra and slip design

The slip, thongs, panties and French as well formed. They different only in the shape of the cut lines to minimize the skin is pressed seam width. Each product shows the selection of the rubber should be carefully followed. The rubber width, thickness and elasticity, and sew technology affect the level of comfort while wearing products.

\subsection{Textilcover design}

The brace material properties take over from the temperature outside, cold in winter, hot in summer. In order to improve the comfort feeling, we make a textile coat to the brace. This solution eliminates the underwear layer. The inside layer of coat is very flexible with good physiological properties, the outer layer is not flexible, insulating properties dimensionally stable, which is assembled and washable.

Only in individual size can be made, taking into account the brace form and design. The coat can be open by zip, easily pulled and removable to brace and easy to wash. (Figure 4)

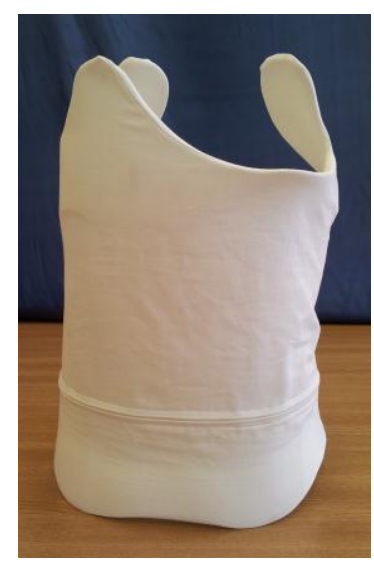

Figure 4: Brace with cover 


\section{Conclusion}

Subjective tests were performed among children who is wearing brace. They tested 5 kinds of raw material sample collections. Their views can be stated that the types of cotton fabrics in certain weather conditions are appropriate in terms of comfort, the PCM-enabled products proved to be the most appropriate in the tested temperature. The brace coated textiles give esthetic and physiological characteristics of the device which can be improved and the vital functions and in the healing effect to devices.

\section{REFERENCES}

1. http://idiopathicscoliosis.com/the-bracing-experience/protocol-of-care.

2. http://www2.sci.u-szeged.hu/fokozatok/PDF/Czibula_Agnes/Czibula_Agnes_Phd_disszertacio.pdf p. 11.

3. Gyimesi S. Textilipari kézikönyv. 1979. p. 53.

4. Árokszállási K. Hôtárolás. A jövő technológiája Roxa Kft. 2002. p. 84.

5. http://www.advancedfibres.eu/coolmax-2/what-is-coolmax/coolmax-comfort-system.

Felina Hungary Rt. give technical assistance to the products preparation.

This work was supported by the project GERINCO2 (TECH_08-A1/2-2008-0121, NDA) respectively. 